\title{
Aquiring High-Quality Microscopic Images Through Silicon Without Damaging the Finished Product
}

\author{
Robert Bellinger ${ }^{1}$ \\ ${ }^{1}$ Product Application Manager, Olympus Corporation of the Americas, Scientific Solutions Group, 48 \\ Woerd Avenue, Waltham, MA 02453, USA.
}

Typical microelectronics failure-analysis protocol requires the ability to inspect circuit patterns through silicon, non-destructively, while maintaining the mechanical integrity of the finished product. Noninvasive techniques such as microscopic imaging in the near-IR spectrum provide inspection directly through silicon. Applications include inspection for short circuits within the product (burnout marks, stress indicators, and so on), bonding alignment (analysis of alignment marks between thinly bonded circuits), inspection after electrical testing (any type of failure), and chip damage (defects in materials, contamination, etc.).

The requirements of most failure-analysis and $\mathrm{R} \& \mathrm{D}$ laboratories for measuring defects, creating reports, and archiving images demand a digital approach. Because of the nature of this application, native contrast is minimal and must be optimized through image-analysis software. Illumination nonuniformity from causes such as vignetting produces darkening of the image corners relative to the center, and must be digitally removed from the live view and captured image.

While reflected-light microscopy is ideal for illuminating a sample from above, transmitted-light IR microscopy brings light to a sample through silicon from beneath the sample, providing higher contrast. The transmitted-light approach is especially useful for the inspection of alignment patterns or fiducial marks through silicon.

The Olympus UIS2 series of dedicated IR objective lenses offers a turnkey solution for near-IR digital imaging. The latest-generation UIS2 dedicated near-IR objective lenses provide increased transmittance at the near-IR spectrum and provide correction collars $(20 \mathrm{x}, 50 \mathrm{x}$, and 100x objective lenses) to be set for specific silicon thickness, maximizing transmittance and performance. The Olympus XM10-IR digital camera provides high-contrast images throughout the near-IR spectrum up to $1100 \mathrm{~nm}$ while still maintaining a large field of view thanks to the large 2/3" CCD. Olympus Stream image analysis software fully controls the XM10-IR camera, unlike other IR imaging solutions, to provide live shading correction to maximize image uniformity across the field of view. Furthermore, the user can easily maximize image contrast on both live and captured IR images. Stream software also provides the ability to perform accurate measurements anywhere within the image field of view, automatically create reports, and archive images and relevant data.

Using shortwave-infrared (SWIR) imaging at longer wavelengths--for example, the 1300-1500 nm range-allows for imaging on more difficult samples such as MEMS devices, heavily doped silicon samples, samples with rough surfaces, wafer bonding, and 3D chip stacks. This method is possible using moresensitive imaging systems such as indium gallium arsenide (InGaAs) cameras. Gaining the signal benefits from dedicated IR objectives, high-power illumination, and InGaAs cameras when using reflected- and transmitted-light microscopy, allowing for imaging on these more difficult samples. 
The latest generation of dedicated near-IR objective lenses can provide increased transmittance at the near-IR spectrum and correction collars (for 20x, 50x, and 100x objective lenses) to be set for specific silicon thickness, maximizing transmittance and performance. Digital cameras can provide high-contrast images throughout the near-IR spectrum up to $1100 \mathrm{~nm}$ while still maintaining a large field of view. And finally, dedicated software can provide live shading correction to maximize image uniformity across the field of view, perform accurate measurements anywhere within the image field of view, automatically create reports, and archive images and relevant data.
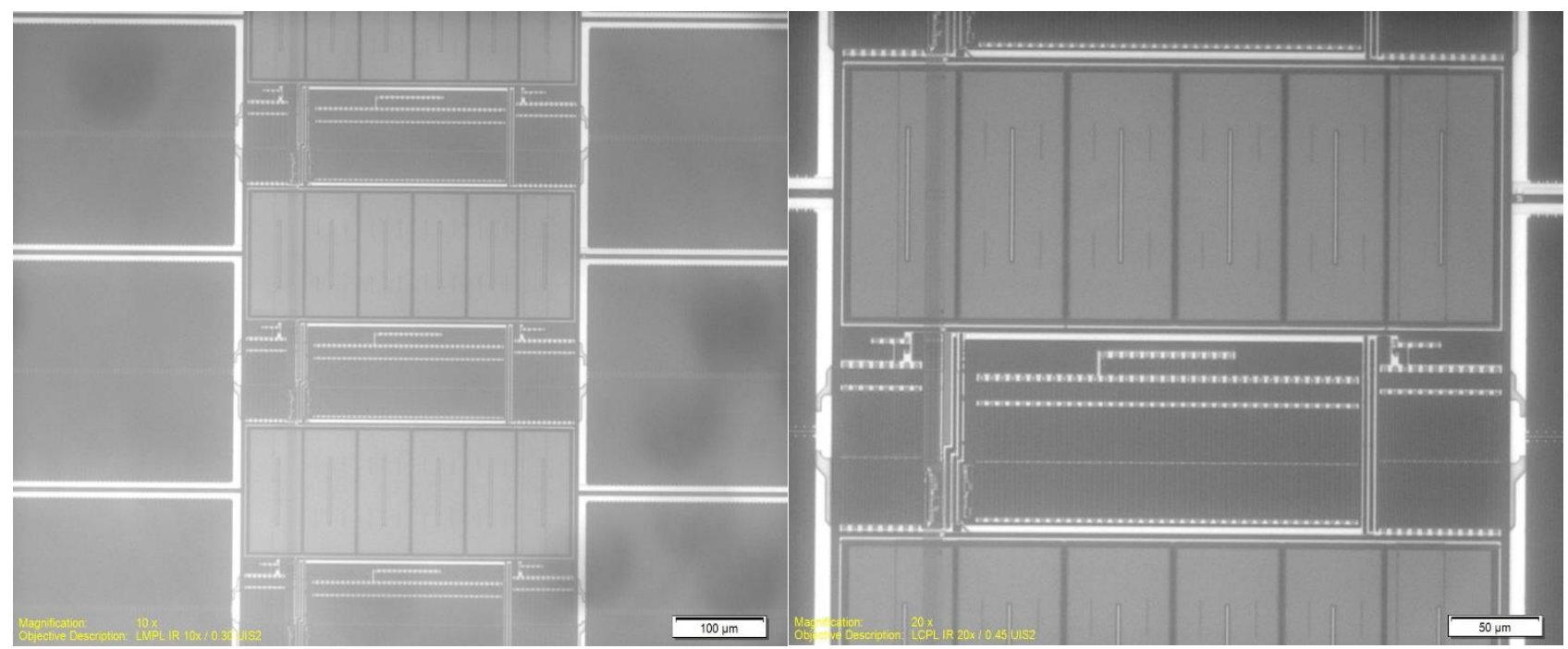

Figure 1. 10x UIS2-IR Objective Lens

Figure 2. 20x UIS2-IR Objective Lens

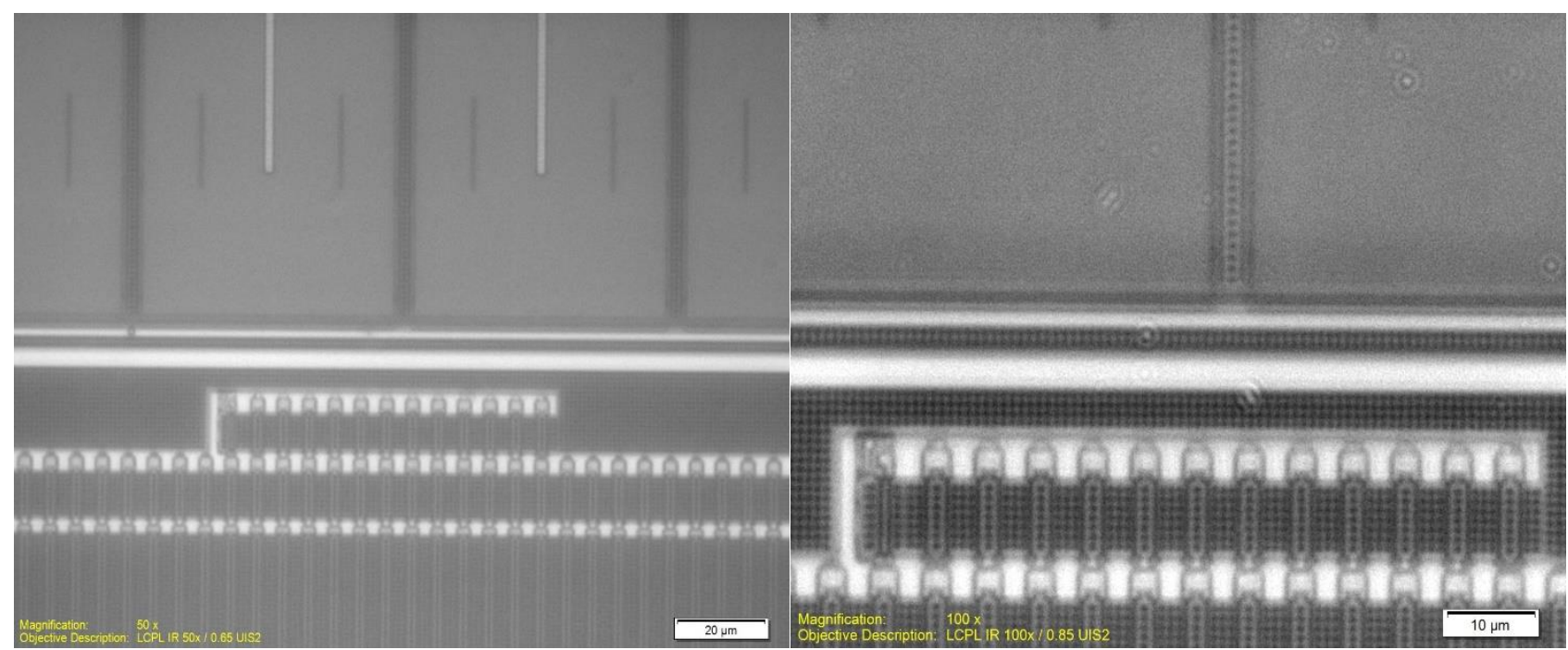

Figure 3. 50x UIS2-IR Objective Lens

Figure 4. 100x UIS2-IR Objective Lens 